\title{
DETERMINATION OF THE CUT-OFF VALUE OF HIGH SENSITIVE C-REACTIVE PROTEIN AND ITS ASSOCIATION WITH DYSLIPIDAEMIA IN SUBCLINICAL HYPOTHYROIDISM
}

\author{
Suparna Roy1, Mohini Gangopadhyay2, Anindya Dasgupta ${ }^{3}$ \\ ${ }^{1}$ Assistant Professor, Department of Biochemistry, Calcutta National Medical College, Kolkata. \\ 2MBBS Student, Calcutta National Medical College, Kolkata. \\ ${ }^{3}$ Professor and HOD, Department of Biochemistry, Calcutta National Medical College, Kolkata.
}

\section{ABSTRACT}

\section{BACKGROUND}

A pro-inflammatory state and dyslipidaemia predispose Subclinical hypothyroidism (SCH) to substantial cardiovascular disorders (CVD). However, there are very less studies on describing a cut-off level of hsCRP as a marker of pro-inflammatory state in SCH beyond which risk factors for CVD become significant.

\section{MATERIALS AND METHODS}

Settings and Design- A hospital based, cross sectional, observational study including 64 SCH cases, 60 overt hypothyroids (OH) and 50 controls. Thyroid hormones and hsCRP were measured by ELISA and immunoturbidimetry respectively.

Statistical Analysis- Cut-off values of hsCRP, TSH and serum cholesterol were assessed by receiver operator and characteristic curve (ROC). Cut-off values of hsCRP between SCH and controls were obtained. Chi square analysis was used to assess the distribution of serum TSH and cholesterol values between the high and low hsCRP patients.

\section{RESULTS}

Higher TSH values showed more significant distribution in the high hsCRP group. Similarly, SCH patients with cholesterol level higher than $170 \mathrm{mg} / \mathrm{dL}$ were found to be more significantly present in the high hsCRP group.

\section{CONCLUSION}

The hsCRP value of higher than $0.81 \mathrm{mg} / \mathrm{L}$ is a significant risk factor for increased risk of dyslipidaemia and CVD in hypothyroid patients as early as in its subclinical state.

\section{KEYWORDS}

Subclinical Hypothyroidism, ROC Curve, hsCRP, Dyslipidaemia.

HOW TO CITE THIS ARTICLE: Roy S, Gangopadhyay M, Dasgupta A. Determination of the cut-off value of high sensitive C-reactive protein and its association with dyslipidaemia in subclinical hypothyroidism. J. Evolution Med. Dent. Sci. 2017;6(28):2315-2320, DOI: $10.14260 / \mathrm{Jemds} / 2017 / 498$

\section{BACKGROUND}

Hypothyroid disorders result from a lack of optimum tissue response to the thyroid hormones due to either their deficient secretion or tissue unresponsiveness due to other causes. It is an endocrinological disorder that plays a major role in affecting human health and morbidity among all noncommunicable diseases. By virtue of its essential role in almost all aspects of metabolism, deficiency of this hormone affects almost all organ systems of our body. Among them cardiovascular system is one of the major cause of thyroid disorder induced morbidity.

Subclinical hypothyroidism (SCH) is an earlier form of hypothyroidism when the transient decreases in the thyroxine levels are compensated by an elevated level of TSH. Overt hypothyroidism $(\mathrm{OH})$ results when this compensatory mechanism fails in the long course of time and the thyroxine levels remain persistently lowered below the reference

Financial or Other, Competing Interest: None.

Submission 18-02-2017, Peer Review 24-03-2017,

Acceptance 30-03-2017, Published 06-04-2017.

Corresponding Author:

Dr. Anindya Dasgupta,

Professor and HOD,

Department of Biochemistry,

Calcutta National Medical College, Kolkata-14.

E-mail: anindya653@gmail.com

DOI: $10.14260 /$ jemds $/ 2017 / 498$ levels. However, the metabolic system starts showing its compromised status since the early stage of $\mathrm{SCH}$. Congruent with this fact, atherosclerosis and immunological changes are reported in both $\mathrm{SCH}$ as well as in $\mathrm{OH}_{(1,2)}$ Keeping in mind that a low-grade inflammatory stage predisposes to the conditions of atherosclerosis through the initiation, progression and stabilisation of the thrombotic plaque, the high sensitivity $\mathrm{C}$ reactive protein (hsCRP) becomes an important predictor of the early low grade inflammation in these patients. It has been found in several studies that hsCRP is a strong independent factor that predicts cardiovascular events, future myocardial infarction, stroke, peripheral arterial disease and vascular health. ${ }^{(3,4)}$ The hsCRP has been found to be increased in hypothyroidism predisposing it to several pro-inflammatory disorders among which CVD is the major one.

Although the relationship between the CVD and overt hypothyroidism is well known for long, evidences are now being reported relating the SCH with CVD along with its associated confounding factors like dyslipidaemia.(5) The hsCRP that indicates a very early rise in the levels of $\mathrm{C}$ reactive protein (CRP) has been found to be elevated in $\mathrm{SCH}$ and linked to early dyslipidaemic changes.(6) Furthermore, the elevated hsCRP level tends to decrease also after substitution of L-thyroxine in hypothyroid patients, is shown by Adamarczuk-Janczyszyn M et al (2016).(7,8) 
However, the level of hsCRP beyond which its increase could be supposed to be associated with hypothyroidism with optimum sensitivity and specificity has not been explored well till date. Keeping in mind the importance of its increase in hypothyroid patients, it was implicated that there is a need for exploration of its cut-off value against increased TSH levels as early as in the SCH stage. Hence, the present hypothesis was made that there may be a cut-off value of hsCRP beyond which it could be attributed to hypothyroidism with significant sensitivity and minimum false positivity as early as in the SCH stage. Accordingly, the present study was designed to find out such a cut-off value and validate it so that it can be used to detect the rise of hsCRP and prevent the related complications at the earliest level.

\section{MATERIALS AND METHODS}

\section{Selection of Study Subjects-}

The present study was conducted as a hospital based, cross sectional, observational study during the period of July 16 to December 16. During this period 110 cases of hypothyroid patients were selected from the thyroid clinic of this institution by the method of convenience. Hypothyroid patients were divided into two groups: (1) SCH with elevated TSH values along with a thyroxine level within its reference range; (2) $\mathrm{OH}$ with elevated TSH and a lowered T4 value below the reference range. 60 patients were included into group 1 while 50 patients were considered in group 2 during our study period. All cases were selected following the method of convenience following the appropriate exclusion and inclusion criteria. This sample size was maximum possible by the method of convenience during the time period of six months with the stipulated inclusion and exclusion criteria. During selection of cases it was kept in mind that none of the cases might suffer from any inflammatory disease, any other endocrine disorders, any malignant disease or any systemic illness that might have an effect on increase in $\mathrm{C}$ reactive protein. Patients having any addiction including smoking, alcoholism or any drug addiction were excluded from the study. Patients with myocardial disease and pregnancy were also not considered in the case group. 50 age and sex matched controls were selected from the same population group with similar socioeconomic and nutritional status. The study protocol strictly adhered to the guidelines of Helsinki declaration for human studies 1975, revised 2000, and the Indian Council for Medical Research. Informed consents were obtained from all participants and the study was initiated after obtaining the written permission from the institutional ethical committee.

\section{Measurement of Study Parameters}

$3 \mathrm{~mL}$ of venous blood was collected following aseptic conditions and was allowed to clot at $370 \mathrm{C}$. After the clotting was complete, the blood samples were centrifuged at 1500 rpm for 5 minutes and the serum was obtained. Haemolysed, icteric and lipaemic samples were rejected. Serum samples were measured immediately for the biochemical assays.

\section{Measurement of Thyroid Profile}

The quantitative determination of TSH and T4 levels in blood were done using a microplate immunoenzymometric assay by ELISA technique. The ELISA kits were obtained from Accubind, USA and the measurement was done using the automated ELISA reader and washer obtained from Tecan, Austria. The tests were validated by using controls for the TSH and T4 within given reference range and the precision was monitored by the coefficient of variation (CV) which was found to be $6.5 \%$ for TSH and $5.5 \%$ for the T4.

\section{Measurement of the hsCRP}

hsCRP methods are highly sensitive and can detect CRP at levels lower than $3 \mathrm{mg} / \mathrm{L}$. In the present study, we used the immunoturbidimetric assay method using the reagent kit CRP-US from USA. It is a turbidimetric immunoassay which is based on agglutination reaction. The test specimen is mixed with latex reagent and activation buffer. The CRP in serum produces an insoluble complex which causes turbidity that is measured by a wavelength between 505 - $578 \mathrm{~nm}$. The increase in turbidity corresponds the conc. of CRP in solution.

\section{Measurement of Lipid Profile}

Lipid profile was measured using the spectrophotometric assay techniques. Total cholesterol was measured by cholesterol oxidase peroxidise method. Both the LDL and HDL cholesterol levels were measured by direct enzymatic methods.

\section{Statistical Calculations}

The data obtained were studied for obtaining their mean values along with standard error of mean (SEM). Relationships between the lipid parameters in the study groups were ascertained by post-hoc ANOVA with Bonferroni correction.

Receiver operating characteristic (ROC) curves were obtained for ascertaining the cut-off values of hsCRP and TSH. Chi square tests and Odds ratio ranges at 95\% confidence interval were performed in $2 \times 2$ contingency tables for validating the cut-off values of TSH by finding out the significance of difference between the two groups of higher hsCRP and lower hsCRP in both SCH and OH group. For all tests $\mathrm{P}$ value was significant at $\mathrm{P}<0.05$ for $95 \%$ confidence interval. For all statistical analyses, the SPSS statistical software for Windows was used.

\section{RESULTS}

\begin{tabular}{|c|c|c|c|}
\hline & SH & OH & Control \\
\hline $\begin{array}{c}\text { Total Cholesterol } \\
\text { Mean/SEM }\end{array}$ & $183 / 4.9$ & $206 / 6.9$ & $162 / 1.9$ \\
\hline LDLc Mean/SEM & $96.2 / 3.7$ & $98 / 2.8$ & $87.6 / 2.6$ \\
\hline HDLc Mean/SEM & $46.9 / 0.9$ & $43.7 / 0.77$ & $50.3 / 1.3$ \\
\hline $\begin{array}{c}\text { Triglyceride } \\
\text { Mean/SEM }\end{array}$ & $117.6 / 8.4$ & $167 / 13.1$ & $112 / 1.9$ \\
\hline \multicolumn{4}{|c|}{$\begin{array}{c}\text { Table 1. Group Statistics Showing the Mean/SEM } \\
\text { of the Lipid Parameters in Different Study Groups }\end{array}$} \\
\hline
\end{tabular}

Data in the Table 1 show the distribution of mean values and their SEs for the lipid parameters in different study groups. It is evident that the total cholesterol and its LDL component is highest for the $\mathrm{OH}$ group followed by that in the SH and control group respectively. In contrary to it, its HDL component shows lowest value in the $\mathrm{OH}$ group followed by that in the SH group. Serum TG followed the same trend as the total cholesterol and its LDL component in all study groups. To ascertain the significance of difference for these 
lipid parameters in different groups ANOVA with Bonferroni correction was performed next.

\begin{tabular}{|c|c|c|c|c|c|}
\hline $\begin{array}{c}\text { Dependent } \\
\text { Variable }\end{array}$ & $\begin{array}{c}\text { (I) } \\
\text { First } \\
\text { Group } \\
\text { in the } \\
\text { pair }\end{array}$ & $\begin{array}{c}\text { (J) } \\
\text { Second } \\
\text { Group } \\
\text { in the } \\
\text { pair }\end{array}$ & $\begin{array}{c}\text { Mean } \\
\text { Difference } \\
\text { between } \\
\text { groups } \\
\text { pairwise } \\
\text { (I-J) }\end{array}$ & $\begin{array}{l}\text { Std. } \\
\text { Error }\end{array}$ & P Value \\
\hline \multirow{6}{*}{$\begin{array}{c}\text { Total } \\
\text { Cholesterol }\end{array}$} & \multirow{2}{*}{ Control } & SH & $-20.09048^{*}$ & 7.56986 & .027 \\
\hline & & $\mathrm{OH}$ & $-43.45714^{*}$ & 8.23763 & .000 \\
\hline & \multirow{2}{*}{$\mathrm{SH}$} & Control & $20.09048^{*}$ & 7.56986 & .027 \\
\hline & & $\mathrm{OH}$ & $-23.36667^{*}$ & 7.26491 & .005 \\
\hline & \multirow{2}{*}{$\mathrm{OH}$} & Control & $43.45714^{*}$ & 8.23763 & .000 \\
\hline & & $\mathrm{SH}$ & $23.36667^{*}$ & 7.26491 & .005 \\
\hline \multirow{6}{*}{$\begin{array}{c}\text { Tri- } \\
\text { Glyceride }\end{array}$} & \multirow{2}{*}{ Control } & $\mathrm{SH}$ & -5.62143 & 13.42135 & 1.000 \\
\hline & & $\mathrm{OH}$ & $-55.82143^{*}$ & 14.60530 & .001 \\
\hline & \multirow{2}{*}{$\mathrm{SH}$} & Control & 5.62143 & 13.42135 & 1.000 \\
\hline & & $\mathrm{OH}$ & $-50.20000^{*}$ & 12.88066 & .000 \\
\hline & \multirow{2}{*}{$\mathrm{OH}$} & Control & $55.82143^{*}$ & 14.60530 & .001 \\
\hline & & $\mathrm{SH}$ & $50.20000^{*}$ & 12.88066 & .000 \\
\hline \multirow{6}{*}{ HDLc } & \multirow{2}{*}{ Control } & $\mathrm{SH}$ & 3.24048 & 1.52883 & .108 \\
\hline & & $\mathrm{OH}$ & $6.55714^{*}$ & 1.66370 & .000 \\
\hline & \multirow{2}{*}{ SH } & Control & -3.24048 & 1.52883 & .108 \\
\hline & & $\mathrm{OH}$ & 3.31667 & 1.46724 & .076 \\
\hline & \multirow{2}{*}{$\mathrm{H}$} & Control & $-6.55714^{*}$ & 1.66370 & .000 \\
\hline & & $\mathrm{SH}$ & -3.31667 & 1.46724 & .076 \\
\hline \multirow{6}{*}{ LDLc } & \multirow{2}{*}{ Control } & $\mathrm{SH}$ & -1.32500 & 4.6654 & 1.00 . \\
\hline & & $\mathrm{OH}$ & -9.91667 & 4.2898 & .047 \\
\hline & \multirow{2}{*}{$\mathrm{SH}$} & Control & 1.32500 & 4.28985 & 1.000 \\
\hline & & $\mathrm{OH}$ & -8.59167 & 4.11703 & .116 \\
\hline & & Control & 9.91667 & 4.66827 & .047 \\
\hline & & $\mathrm{SH}$ & 8.59167 & 4.11703 & .116 \\
\hline \multicolumn{6}{|c|}{$\begin{array}{l}\text { Table 2. ANOVA with Bonferroni Correction to show } \\
\text { the Significance of Difference of Different Parameters } \\
\text { of Lipid Profile pairwise between Different Study Groups }\end{array}$} \\
\hline
\end{tabular}

$\mathrm{P}$ value was considered to be significant at $\mathrm{P}<0.05$ for a 95\% confidence interval.

The post-hoc ANOVA analysis revealed the significance of variations of the different components of total serum cholesterol and triglyceride in the subclinical and overt stages of hypothyroidism. For serum cholesterol, the high value in the $\mathrm{OH}$ group was statistically significant compared to that found in both the $\mathrm{SH}$ and control groups. The value in the SH group was also significantly higher than that in the control group. Same trend was observed also in for the serum triglyceride values. However, the values of the LDLc and HDLc although showed significantly higher and lower values respectively in the $\mathrm{OH}$ groups compared to the control groups, the difference was not significant when compared to the SCH group.

For finding the cut-off value of the hsCRP between the hypothyroid and euthyroid groups, ROC curve analysis was done. From it the cut-off value of the hsCRP was ascertained to be 0.81 against a sensitivity of 0.786 and specificity of 0.323 .

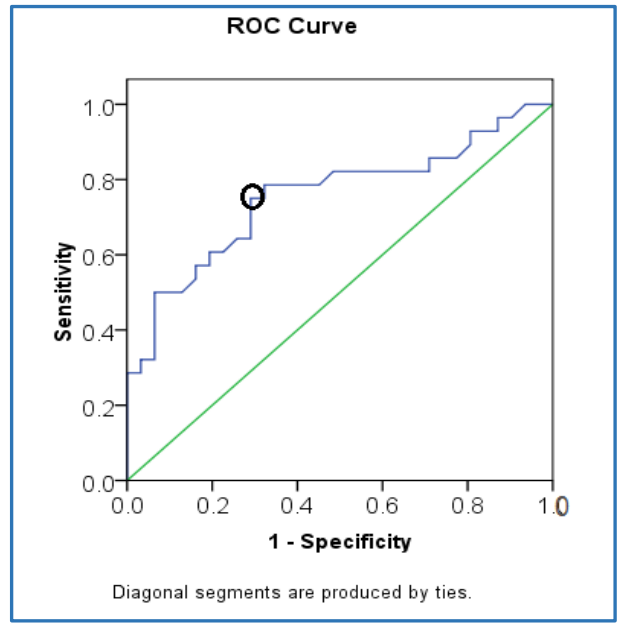

Figure 1. ROC Curve Showing the Cut-off Value of hsCRP

In the given ROC analysis with an area under curve (AUC) of 0.755 , the cut-off value of the hsCRP was ascertained to be 0.81 against a sensitivity of 0.786 and specificity of 0.323 .

For finding the significance of the distribution of hypothyroid and euthyroid subjects above and below this cut-off value of hsCRP, we determined the cut-off value of TSH between the euthyroid and hypothyroid subjects in our setup.

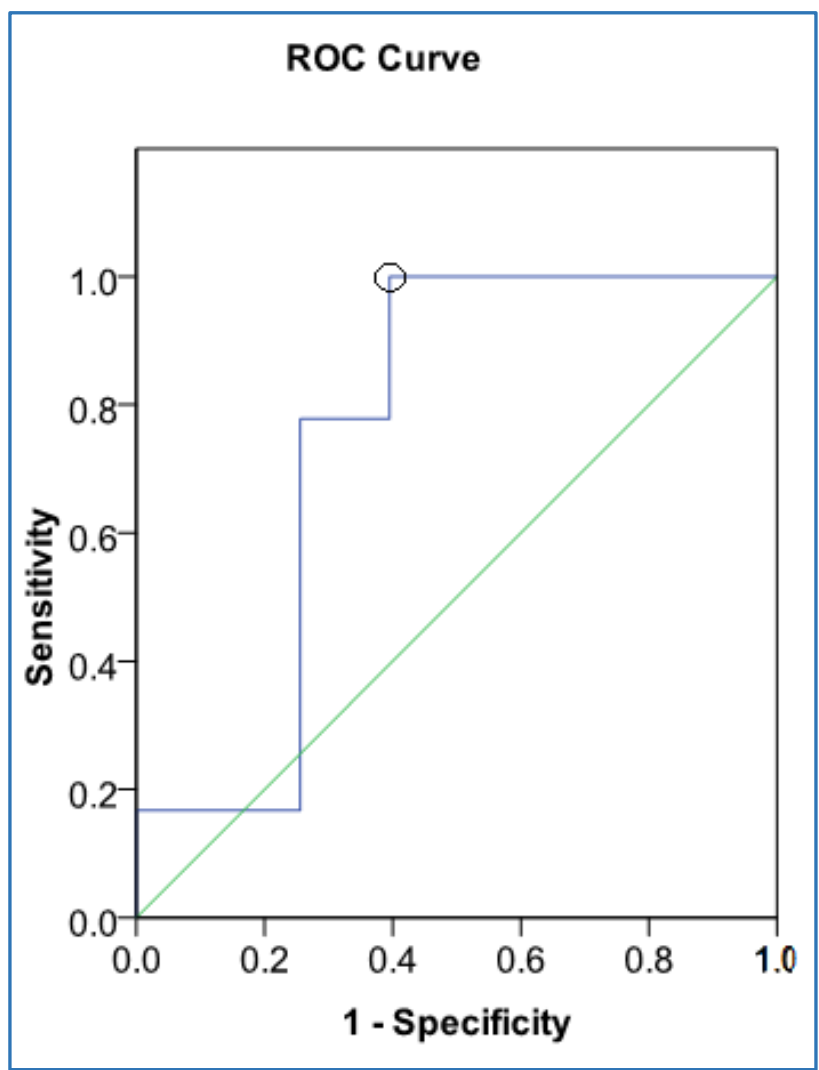

Figure 2. ROC Curve Showing the Cut-off Value of Serum TSH

Figure 2. ROC curve showing the cut-off value of serum TSH which is 6.6 against a sensitivity 0.944 and false positivity of 0.395 as evident from the ROC having an AUC of 0.756 . This value is close to the cut-off value of 6.3 provided in the ELISA reagent kit (Accubind USA) used by us. 
After ascertaining the cut-off values of the hsCRP and TSH values, the significance of difference in distribution of patients having TSH values higher and lower than $6.6 \mathrm{mIU} / \mathrm{L}$ in both high and low hsCRP was evaluated by chi square and odds ratio range for $95 \%$ confidence interval in both $\mathrm{SCH}$ and $\mathrm{OH}$ groups separately.

\begin{tabular}{|c|c|c|}
\hline & hSCRP $>0.81$ & hSCRP $<0.81$ \\
\hline TSH $\geq 6.6$ & 25 & 6 \\
\hline TSH $<6.6$ & 18 & 15 \\
\hline $\begin{array}{c}\text { Table 3. Chi Square and Odds Ratio for the Distribution } \\
\text { of Higher and Lower Values of TSH than } 6.6 \text { mIU/L } \\
\text { in the SH Group }\end{array}$ \\
\hline
\end{tabular}

$\chi 2$ and OR show the significance of distribution of patients having TSH $>6.6$ and $<6.6$ among those having the hsCRP levels higher and lower than the cut-off value of 0.81 as calculated in the $\mathrm{SH}$ group in the present study. The value of $\chi 2$ is 4.94 \& OR is 3.47 with a range of 1.21 to 17.7 for a $95 \%$ confidence interval.

\begin{tabular}{|c|c|c|}
\hline & hsCRP $>0.81$ & hsCRP $<0.81$ \\
\hline TSH $\geq 6.6$ & 28 & 3 \\
\hline TSH $<6.6$ & 3 & 7 \\
\hline
\end{tabular}

Table 4. Chi Square and Odds Ratio for the Distribution of Higher and Lower Values of TSH than 6.6 in the OH Group

$\chi 2$ and OR show the significance of distribution of patients having TSH $>6.6$ and $<6.6$ among those having the hsCRP levels higher and lower than the cut-off value of 0.81 as calculated in the $\mathrm{OH}$ group in the present study. The value of $\chi 2$ is $14.91 \&$ OR is 21.7 with a range of 4.65 to 101.7 for a $95 \%$ confidence interval.

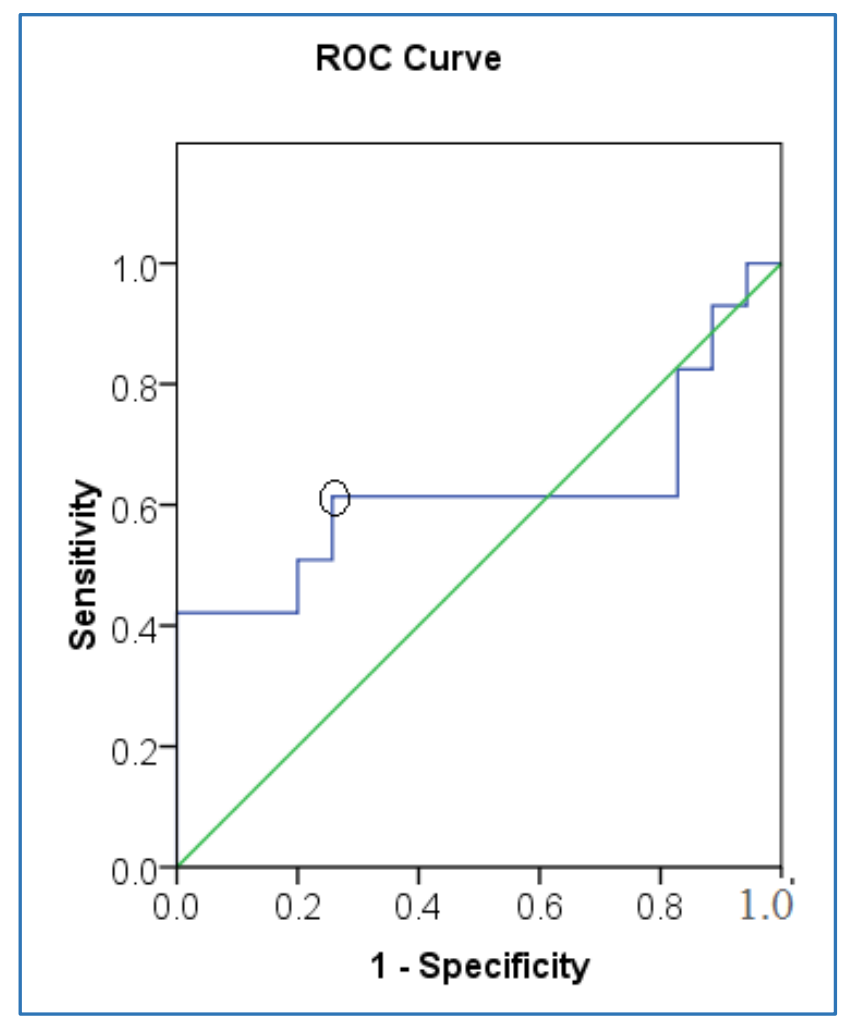

Figure 3. ROC curve Showing the Cut-off value of Serum Cholesterol between the SCH and Control Groups
Figure 3. ROC curve showing the cut-off value of serum cholesterol between the SCH and control groups. With an AUC of 0.622 , the cut-off value is seen to be $170 \mathrm{mg} / \mathrm{dL}$ with a specificity of 0.614 and a false positivity of 0.257 .

\begin{tabular}{|l|c|c|}
\hline & $\begin{array}{c}\text { Cholesterol } \\
\mathbf{2 1 7 0} \mathbf{~ m g} / \mathbf{d L}\end{array}$ & $\begin{array}{c}\text { Cholesterol } \\
<\mathbf{1 7 0} \mathbf{~ m g} / \mathbf{d L}\end{array}$ \\
\hline $\mathrm{HsCRP}>0.81 \mathrm{mg} / \mathrm{L}$ & 22 & 10 \\
\hline $\mathrm{HsCRP}<0.81 \mathrm{mg} / \mathrm{L}$ & 9 & 17 \\
\hline
\end{tabular}

Table 5. Chi Square and Odds Ratio for the Distribution

of Higher and Lower Total Cholesterol Values in

Persons having hsCRP Values more than $0.81 \mathrm{mg} / \mathrm{L}$

Chi sq $=6.72, \mathrm{P}=0.009$

$\mathrm{OR}=4.6$, Range $=1.38$ to 12.50 at $95 \%$ confidence interval.

\section{DISCUSSION}

The present study was undertaken to ascertain a cut-off value of hsCRP beyond which its increase could be attributed to hypothyroidism with maximum sensitivity with minimum false positivity. At first, we decided to obtain a cut-off value for TSH to differentiate between the hypothyroid and euthyroid patients in our population in a better realistic way. For this we used the ROC curve analysis of the TSH values including both cases and controls and found the optimum cut-off value of TSH to be $6.6 \mathrm{mIU} / \mathrm{L}$ with a maximum possible sensitivity 0.944 against a minimum false positivity of 0.395 (Figure 2). Thereafter, according to the obtained cutoff mark of TSH in the present study, the study subjects were again divided into euthyroid and hypothyroid cases. Hypothyroid patients were further divided into the SCH and OH group on the basis of TSH and T4 values. When Chi square value and the odds ratio were calculated between the high hsCRP and low hsCRP groups in euthyroid, SCH and $\mathrm{OH}$ groups, the difference was statistically significant (Table 3 and 4). Chi square values of 4.84 and 14.91 along with odds ratio ranges more than 1 for both $\mathrm{SCH}$ and $\mathrm{OH}$ separately indicated that the cut-off value of $0.81 \mathrm{mg} / \mathrm{L}$ for the hsCRP was effective in differentiating between both of the SCH and $\mathrm{OH}$ groups from the euthyroid patients. In other words, both the $\mathrm{SCH}$ and $\mathrm{OH}$ group had their hsCRP values more than 0.81 $\mathrm{mg} / \mathrm{L}$.

Increased value of hsCRP is closely linked to cardiovascular complications in both SCH and $\mathrm{OH}$. Thyroid hormone has genomic and nongenomic effects on heart and systemic vasculature. The genomic action regulates intracellular calcium cycling and formation of contractile protein in cardiac myocytes.(9) The nongenomic events include regulation of $\mathrm{Na}^{+}-\mathrm{K}^{+}$ATPase pump, action polymerisation and adenine nucleotide translocator 1 in the mitochondrial membrane.(10) These effects in turn play a role in regulation of cardiac function and cardiac haemodynamics.

It is reported that in hypothyroidism there is abnormality of endothelial inflammation and hsCRP is elevated due to interaction of IL- 6 on TNF $\alpha$ and IL-1.(11) Some recent studies also reported an increased hsCRP values in the SCH stages,(8) but none has so far described a cut-off value for this proinflammatory cytokine in the hypothyroid disorders. In our study, the cut-off value of $0.81 \mathrm{mg} / \mathrm{L}$ for the hsCRP implies that in hypothyroidism as early as its subclinical stage hsCRP value higher than this potentiates the risks associated with proinflammatory conditions including CVD. 
Dyslipidaemia has been a close confounding factor for hypothyroidism. Thyroid hormones not only upregulate the LDL receptors, but they also control the expression of sterol regulatory element-binding protein 2 (SREBP 2) and prevent LDL from oxidation.(12,13) It also regulates HDL metabolism by increasing cholesteryl ester transfer protein activity(CETP).(14) Thyroid hormone also activates lipoprotein lipase and hepatic lipase and helps in conversion of LDL to small dense LDL.(15-17) Thyroid hormone helps in TG metabolism by upregulating the expression of apolipoprotein AV (Apo AV).(18)

Increased hsCRP has been associated with increased risk of dyslipidaemia and CVD in both SCH and OH. To explore the dyslipidaemic changes in hypothyroid patients, lipid profile was estimated in both cases and controls. Serum cholesterol was found to be highest in the $\mathrm{OH}$ group followed by that in the SCH and normal euthyroids as evident by ANOVA (Table 2). These signified that increase in serum cholesterol level in hypothyroid disorders start as early as its subclinical phase. Several reasons like defects in LDL receptors, a reduced inhibition of HMG CoA reductase activity, etc account for increased cholesterol levels in hypothyroidism. However, in the present study, the increase in LDLc and reduction in HDLc was significantly evident in $\mathrm{OH}$ groups only. Although the mean values of LDLc and HDLc were respectively higher and lower in the SH group also in comparison to the healthy controls, but this difference was not statistically significant reflecting an early stage of their deviation from the normal values. To assess the increased serum cholesterol level with elevated hsCRP values at the early stages of hypothyroidism, we derived a cut-off value of serum cholesterol of $170 \mathrm{mg} / \mathrm{dL}$ using the ROC (Figure 3) between the SCH and control group. We distributed the serum cholesterol values of SCH patients above and below its obtained cut-off level into two groups of high and low hsCRP followed by analysing their difference by Chi square analysis (Table 5). The association of elevated hsCRP with hyperlipidaemia is evident through the results shown in the Table 5 where a Chi square value of 6.72 with a $P$ value of 0.009 and an odds ratio of 4.6 (Range of 1.38 to 12.5 at a $95 \%$ confidence interval) indicates that SCH patients having a cholesterol value more than $170 \mathrm{mg} / \mathrm{dL}$ are significantly more found in the SCH group having the hsCRP level more than $0.81 \mathrm{mg} / \mathrm{L}$. These findings clearly indicate the potential link between hypercholesterolaemia and high hsCRP values in hypothyroid cases.

Considering all these findings regarding the increase in hsCRP level and serum cholesterol level, it can be suggested from the present study that in spite of a clinical silence, inflammatory and metabolic derangements are initiated in the subclinical stage of hypothyroidism when serum hsCRP values rise beyond $0.81 \mathrm{mg} / \mathrm{L}$. This may be used to assess and monitor the early changes of the important cytokine hsCRP starting from the early stages of $\mathrm{SH}$.

\section{CONCLUSION}

The pro-inflammatory conditions that prevail in the subclinical stage of hypothyroidism are important contributors for cardiovascular and metabolic risks. Our study evaluated a cut-off value of TSH which can be utilised for identifying the pro-inflammatory status and manage them accordingly that can substantially reduce the cardiovascular mortality and morbidity. Thus, patients with hsCRP value above $0.81 \mathrm{mg} / \mathrm{L}$ should be monitored for dyslipidaemia and other CVD risk factors to prevent further complications at its earliest.

\section{ACKNOWLEDGEMENT}

This work is a part of the project sanctioned by the Indian Council of Medical Research (ICMR) as an STS project. We acknowledge ICMR for their sanctioning of the project.

\section{REFERENCES}

[1] De Miranda PEJ, Bittencourt MS, Pereira AC, et al. Subclinical hypothyroidism is associated with higher carotid intima-media thickness in cross-sectional analysis of the Brazilian Longitudinal Study of Adult Health (ELSA-Brasil). Nutr Metab Cardiovasc Dis 2016;26(10):915-21.

[2] Marchiori RC, Pereira LA, Naujorks AA, et al. Improvement of blood inflammatory marker levels in patients with hypothyroidism under levothyroxine treatment. BMC Endocr Disord 2015;15:32.

[3] Yu H, Rifai N. High-sensitivity C-reactive protein and atherosclerosis: from theory to therapy. Clin Biochem 2000;33(8):601-10.

[4] Jialal I, Devaraj S. Inflammation and atherosclerosis: the value of the high-sensitivity C-reactive protein assay as a risk marker. Am J Clin Pathol 2001;116 Suppl:S108-15.

[5] Roy S, Banerjee U, Dasgupta A. Effect of sub clinical hypothyroidism on C-reactive protein and ischemia modified albumin. Mymensingh Med J 2015;24(2):379-84.

[6] Roy S, Banerjee U, Dasgupta A. Interrelationship of the pro-inflammatory marker HSCRP with dyslipidemic changes: a comparative study between subclinical and overt hypothyroidism. J Evolution Med Dent Sci 2016;5(16):806-12.

[7] Adamarczuk-Janczyszyn M, Zdrojowy-Welna A, Rogala $\mathrm{N}$, et al. Evaluation of selected atherosclerosis risk factors in women with subclinical hypothyroidism treated with L-Thyroxine. Adv Clin Exp Med 2016;25(3):457-63.

[8] Yu YT, Ho CT, Hsu HS, et al. Subclinical hypothyroidism is associated with elevated highsensitive C-reactive protein among adult Taiwanese. Endocrine 2013;44(3):716-22.

[9] Rutigliano G, Zucchi R. Cardiac actions of thyroid hormone metabolites. Mol Cell Endocrinol 2017:7.

[10] Koromilas C, Liapi C, Zarros A, et al. Inhibition of $\mathrm{Na}(+), \mathrm{K}(+)$-ATPase in the hypothalamus, pons and cerebellum of the offspring rat due to experimentallyinduced maternal hypothyroidism. J Matern Fetal Neonatal Med 2015;28(12):1438-44.

[11] Akinci B, Comlekci A, Yener S, et al. The alteration of serum soluble CD40 ligand levels in overt and subclinical hypothyroidism. Hormones (Athens) 2007;6(4):327-33.

[12] Shin DJ, Osborne TF. Thyroid hormone regulation and cholesterol metabolism are connected through Sterol Regulatory Element-Binding Protein-2 (SREBP-2). J Biol Chem 2003;278(36):34114-8. 
[13] Faure P, Oziol L, Artur Y, et al. Thyroid hormone (T3) and its acetic derivative (TA3) protect low-density lipoproteins from oxidation by different mechanisms. Biochimie 2004;86(6):411-8.

[14] Lagrost L. Regulation of cholesteryl ester transfer protein (CETP) activity: review of in vitro and in vivo studies. Biochim Biophys Acta 1994;1215(3):209-36.

[15] Kuusi T, Saarinen P, Nikkila EA. Evidence for the role of hepatic endothelial lipase in the metabolism of plasma high density lipoprotein2 in man. Atherosclerosis 1980;36(4):589-93.
[16] Santamarina-Fojo S, Gonzalez-Navarro H, Freeman L, et al. Hepatic lipase, lipoprotein metabolism and atherogenesis. Arterioscler Thromb Vasc Biol 2004;24(10):1750-4.

[17] Rizos CV, Elisaf MS, Liberopoulos EN. Effects of thyroid dysfunction on lipid profile. Open Cardiovasc Med J 2011;5:76-84.

[18] Prieur X, Huby T, Coste H, et al. Thyroid hormone regulates the hypotriglyceridemic gene APOA5. J Biol Chem 2005;280(30):27533-43. 